\title{
Palliation and the caring hospital - filling the gap
}

PA Levack

Retired Consultant in Palliative Medicine and previously Principal in General Practice, Dundee, UK

KEYWORDS Palliative care, hospital

Correspondence to PA Levack

DECLARATIONS OF INTERESTS No conflicts of interest declared.

Little Court

69 Dundee Rd

Dundee DD5 INA, UK

tel +44 (0) I382 779/60

email plevack@btinternet.com

\section{THE PROBLEM OF PALLIATIVE CARE IN HOSPITAL}

Palliation literally means comfort for the ill, but many in the general public as well as health professionals don't understand exactly what palliative care is. The most widely held (and historical) view is that it is care at the very last stage of life, around the time of death. However, palliative care as more recently defined is moving away from treating just the imminently dying to those patients with any serious or life-threatening illness, who need relief from symptoms, pain or distress, at any point from diagnosis to death.' While this much broader definition is a long way from the original idea of terminal care, it unquestionably includes it. It is easy to get 'stuck' discussing definitions of palliative care, but there is greater agreement about its goals: a shift in focus from cure to palliation and an emphasis on maximising quality of life. This involves experienced, skilled management of pain and other distressing symptoms; expertise in acknowledging when a patient may be dying; and a willingness to discuss what is happening with the patient and their family and helping them make plans for future care.

None of these goals are unique to palliative care. It is unhelpful to think of it as the antithesis of the rest of hospital care; aggressive chemotherapy or surgical by-pass of an obstructed malignant bowel can provide good palliation under certain circumstances and doctors in many specialties provide care to alleviate symptoms and address concerns with no expectation of cure.

Patients with advanced illness don't want to choose between aggressive disease management and comfort care - they generally want both. Palliative care specialists therefore need to work closely with other specialists to improve their joint understanding of the complexities at this stage of illness and to provide patients with the benefits of their combined expertise. This is particularly important when treatments may not be working, symptoms are becoming more troublesome and when the benefits and burdens of different treatment approaches are increasingly difficult to balance with the hopes and wishes of the patient.

Hospices provide an outstanding specialist service for those who are able to access it. Due to the success of the hospice model in the UK, there has been for some time an assumption that this type of care will be available for all those who need it; however, in reality fewer than one patient in 20 dies in a hospice (in Scotland, 55\% patients die in hospital and $5 \%$ in a hospice ${ }^{2}$ ).

Patients in the acute setting are often more complex, with more disciplines involved and more clinical uncertainty about prognosis. As $50 \%$ of hospital complaints relate to end-of-life care, ${ }^{3}$ it is clear there is a considerable unmet palliative care need. ${ }^{4}$ Patient and family accounts of unnecessary suffering reached such a magnitude in 2013 that the UK government requested independent reviews of hospital care (Mid Staffordshire Report) $)^{5}$ and of end-of-life care (More Care Less Pathway). ${ }^{6}$

In short, there is irrefutable evidence of a serious gap in hospital care for patients with palliative care needs. This is partly due to the numbers of patients involved; many more patients die in a hospital than in a hospice, ${ }^{7}$ and this number is predicted to increase, perhaps by $20 \%$ by $2030,{ }^{8}$ despite aspirations to support more patients dying at home. It is also due to the present balance of services, with little clinical, governmental or financial focus on palliative care provision in hospitals.

The problems associated with palliative care are also related to the model of delivery. It is widely believed that ward staff should provide most of the palliative care needs in a hospital setting (except when specialist help is required). However, many nurses and doctors working in hospitals focused on cure and hope for cure often feel ill-equipped to look after patients who are very ill and may be dying. ${ }^{9}$ This is especially difficult when they also provide care for those receiving active treatment for acute illness. Cure and care can appear incompatible, especially in an environment where doctors and nurses are under daily pressure to meet treatment targets. The result is often a failure to openly acknowledge that a patient is dying. Furthermore, despite the frequency of death in a hospital, few junior medical staff have clinical exposure to patients who are dying before they start on the wards. ${ }^{10}$ The recent Shape of Training report underlines the need to broaden training in order to better meet patients' needs." 
There is a pressing clinical and moral duty for hospitals to provide a skilled and structured palliative care service $^{12}$ and to take seriously the experiences of patients and their families. Proof of palliative care's effectiveness will be reflected in a tolerable quality of life for patients (and families) during the late stages of their illness. For many patients this includes getting home from hospital.

\section{THE NEED FOR THIS CARE IN HOSPITALS}

Patients with serious illnesses have complex and changing needs. One-third of hospital beds are occupied by patients in their last year of life, ${ }^{13}$ and most hospital deaths are not sudden but follow a documented period of decline. At any one time in a hospital up to $35 \%$ of all inpatients may have palliative care needs affecting their quality of life. ${ }^{14,15}$ The commonest complaint from patients and their families is poor pain control and delayed analgesia, yet knowledge and guidelines about pain control have been available for more than 30 years. ${ }^{16}$ The problem is less a lack of knowledge, but a lack of confidence and a more deeply rooted concern about the [over]use of opioids. ${ }^{17}$

It is unclear how many patients have conditions that are complex enough to require specialist advice in palliative care, but in hospitals where this is available, approximately $20 \%$ or one in five are referred, ${ }^{18}$ although this may be an underestimate of those who would benefit. ${ }^{19}$ Of those referred, it is estimated that $20 \%$ would benefit from more intensive inpatient palliative care. The World Health Organization public health strategy to incorporate palliative care into existing health systems recommends that $30 \%$ of palliative care beds should be in hospital. ${ }^{20}$ How did we reach this point? There are a number of reasons.

\section{Lack of agreement on definitions of palliative care}

Much specialty time has been occupied discussing definitions, despite the fact that many have little or no meaning for patients and their families. If staff, patients and families do not understand what could be available, they do not know what to ask for and when. Perhaps we should simply view palliative care from the perspective of the patients and their families, as an extra layer of available support (Table I). ${ }^{21}$

\section{The success of the community model}

NHS hospices are small and physically (and culturally) separate from an acute hospital. Such separateness allows control of their boundaries and values, i.e. restrict and decide who is admitted and they can practice independently in some ways from the mainstream NHS. In contrast hospitals are large and complex collections of specialist units with increasingly specialised staff, who cannot be assumed to have a broad range of skills outside their specialty. Palliative care faces a challenge in
TABLE I An understandable description of palliative care (Public opinion research in $20 \mathrm{II}$ by the Centre to Advance Palliative Care, www.capc.org)

Palliative care is specialised medical care for people with serious illnesses. This type of care is focused on providing patients with relief from the symptoms, pain and stress of a serious illness - whatever the diagnosis.

The goal is to improve quality of life for both the patient and the family. Palliative care is provided by a team of doctors, nurses and other specialists who work with a patient's other doctors to provide an extra layer of support. Palliative care is appropriate at any stage in a serious illness, and can be provided together with curative treatment

TABLE 2 Difficulties providing palliative care in a hospital'2

The number of specialties and conflicting priorities e.g. waiting time targets, increased usage of fewer beds, pressure to maintain discharge rates.

Due to the increase in potential interventions in previously untreatable illnesses, the interface between medical, oncological or surgical palliative treatment and supportive palliative treatment is complex, although both are nearly always necessary

Recognition of dying can be very difficult or indeed impossible when the clinical picture is uncertain or changing.

Physicians in different specialties can find specific aspects of providing palliative care difficult in the context of their specific area of expertise.

Delivering education with a high turnover of staff is a challenge Lack of specialist palliative care staff.

the hospital setting (Table 2), and it is unrealistic to expect that the hospice model will or should readily transfer to an acute setting.

\section{HOSPITAL PALLIATIVE CARE IN THE UK}

\section{Specialist palliative care advisory services}

The most common model in the UK is the hospital specialist palliative care team which offers advice to the patient's consultant and ward staff, but does not have overall responsibility for patient care. Palliative care teams work with a range of hospital nurses and doctors who may be unfamiliar or uncomfortable with the care of patients who might be dying.

A typical team is small, consisting of one to four specialist palliative care nurses and one or two senior palliative care doctors for hospitals with up to 800 beds. Specialist palliative care nurses usually provide a weekday office hours service and palliative care consultants are often attached to the local hospice and visit as required. For most of the week, therefore, there is no on-site specialist doctor in palliative care. 
Pain and symptom management, the cornerstone of palliative care, can be very complex and require considerable expertise. Symptoms often intensify rapidly and demand confident, skilled diagnosis and management. Discussions about emotive issues of life and death also require considerable skill. It has been suggested that at least 10,000 hours of practice are required to achieve a level of mastery of anything. ${ }^{22}$ Such levels of expertise in palliative care are likely to require in excess of 10,000 hours - the equivalent to six years of full-time working.

\section{Dedicated hospital palliative care beds and units}

In her review of the Liverpool Care Pathway (LCP) and wider issues related to hospital palliative care, Baroness Neuberger singled out the potential for specialist units or dedicated beds to positively influence care and culture in hospitals.

There could be considerable advantages to offering this dedicated service and this plan should be considered, even if it requires devising new financial models to enable it. These areas could not only improve end-of-life care but would also enable hospital staff to gain additional expertise and experience in caring for the dying, having difficult conversations and working with bereaved relatives. An adequately resourced specialist palliative care service which can act as a model of good practice and a hub for maintaining competencies within each hospital would do much to raise standards. ${ }^{6}$

\section{Palliative care wards}

In Scotland, four hospitals have palliative care wards or units. The Royal Alexandra Hospital in Paisley has a 12-bedded surgical palliative care ward. Patients remain under the care of their consultant surgeon, with specialist palliative care team input as necessary. It is neither a hospice ward nor a specialist palliative care unit as it is not staffed by specialist palliative care doctors and nurses. However, its location, in the main hospital, has encouraged discussion and diffusion of a palliative care approach for patients with serious illness. Both foundation level doctors and specialist registrars spend time there as part of their surgical training.

The Queen Margaret Hospital in Dunfermline has a ward that is a combination of an acute palliative care unit and a hospice ward. As a busy diagnostic and treatment centre it cares for more 'acute' patients than a hospice.

The Alexandra Unit in Dumfries Royal Infirmary has eight beds in single rooms. It was purpose-built as a hospice unit but functions as a hospital specialist palliative care unit. Staff provide advice and assessment for the whole hospital, from paediatrics to care of the elderly and outpatient assessment at outpatient and oncology clinics.
Ninewells Teaching Hospital and Cancer Centre in Dundee has a dedicated, short-stay acute palliative care unit of three beds (single side rooms) in a surgical ward. Established with charitable funding, a palliative medical consultant is responsible for patient care, supported by the palliative care team and ward nurses with some palliative medicine training. It provides intensive palliative care for patients with the most complex needs. ${ }^{24} \mathrm{It}$ is supported by other services such as pharmacy, physiotherapy, anaesthesia (using, for example, epidural analgesia), spiritual care and complementary therapy. Foundation year doctors rotate through the ward, which includes breast surgery, plastic surgery, burns and palliative care. Patients can access palliative care and symptom control 24 hours a day, seven days a week. It is also an on-site resource for research and training.

\section{SOLUTIONS FOR IMPROVING PALLIATIVE CARE FOR PATIENTS IN HOSPITAL}

\section{Expanding the hospice model}

Many more hospices are required in order to provide care for those who need it but this would not solve the problem; most patients who are ill need to be admitted to hospital first, for tests and treatment. In addition, when so many treatments and interventions are possible, it can be difficult to be certain when treatment will no longer help and the prospect of recovery is diminishing. By then, it may be too late, the patient may be too unwell to transfer to a hospice or unwilling to leave the security of the hospital and the staff they know. The staff may also feel a responsibility to continue to care for the patient.

\section{Expanding hospital palliative care teams}

Hospital palliative care teams have a limit to their effectiveness, defined by their advisory role and their limited working hours. One in five patients referred are likely to have sufficiently complex needs to require a more intensive approach, with an extended range of specialists closely involved with their care.

\section{Transferring hospice skills into hospital}

The effective transfer of learning from a hospice with 20-30 beds (the size of a hospital ward), to a busy several hundred-bedded hospital, managed, funded and organised along completely different lines, needs to be approached with care, and prospective data need to be carefully collected. The results of 'rolling out' the LCP are a potent example of what can go wrong, despite the best of intentions.

\section{An integrative approach}

Embedding a palliative care service into the culture and practice of a busy acute hospital is a challenge (Table 2). Hospice care and hospital care each have distinct strengths (Table 3), but neither model alone can provide adequate palliative care for patients when they are in hospital. 
TABLE 3 Advantages of hospital, hospice and integrative models for palliative care

\begin{tabular}{|l|l|l|}
\hline \multicolumn{1}{|c|}{ Hospital care } & \multicolumn{1}{|c|}{ Hospice care } & \multicolumn{1}{c|}{ Integrative care } \\
\hline $\begin{array}{l}\text { Access to many specialties, } \\
\text { treatments and investigations }\end{array}$ & Usually not on site & Yes \\
\hline $\begin{array}{l}\text { NHS/university interface: support } \\
\text { and involvement research }\end{array}$ & Limited & Yes - much needed \\
\hline $\begin{array}{l}\text { Long-term relationship with patients } \\
\text { in some specialties, e.g. renal, } \\
\text { chronic respiratory, many cancers }\end{array}$ & Often lost & $\begin{array}{l}\text { Yes. Patients' 'own doctor' can still be } \\
\text { involved with their care }\end{array}$ \\
\hline \multirow{5}{*}{} & Public understand and value hospice & Yes - in models so far \\
\cline { 2 - 3 } & Quiet environment with good facilities & $\begin{array}{l}\text { Probably better than general ward. } \\
\text { Not perfect but on-site }\end{array}$ \\
\cline { 2 - 4 } & 24-hour access to expertise in palliative care & Yes \\
\cline { 2 - 3 } & $\begin{array}{l}\text { Patients know the stage of their illness } \\
\text { and understand that further treatment } \\
\text { will be largely symptomatic }\end{array}$ & $\begin{array}{l}\text { Yes, although 'active treatment' e.g. } \\
\text { chemotherapy, radiotherapy, dialysis, may } \\
\text { still be appropriate }\end{array}$ \\
\cline { 2 - 3 } & Staff working towards same goal of care & $\begin{array}{l}\text { May not always be so clear - but that is the } \\
\text { complex area hospital palliative care works in }\end{array}$ \\
\cline { 2 - 3 } & Effective community links & Yes - helps patients go home \\
\cline { 2 - 3 } & Professional time for family & $\begin{array}{l}\text { Pool of specialist staff to spread expertise } \\
\text { throughout the hospital }\end{array}$ \\
\cline { 2 - 3 } & &
\end{tabular}

It is often said the 'hospital culture' has to change, but if the benefits of hospice care could be integrated without cancelling out the benefits of hospital care, a new model could be established. The resulting integrative model would be a creative combination of two different models, as described by Martin in The Opposable Mind. ${ }^{25}$

\section{An integrative model of hospital palliative care}

As Ballat and Campling wrote in their book Intelligent Kindness - if we acknowledge the gap in care, we have to do something about it. ${ }^{26}$ An integrative model (Table 3) will retain the benefits of a hospital, but will not equal hospice standard. Patients who would benefit from specialist palliative care input can have this on-site. All hospitals and inpatient populations are different, therefore solutions will be different. In one model with dedicated beds, staffed by palliative care trained nurses and doctors, the results showed fast, effective symptom control, rapid discharge home and high patient and family satisfaction for patients with the most complex palliative care needs. ${ }^{24}$ When patients with these complex needs have dedicated beds, they are more visible to staff, who can see for themselves what palliative care does, and how it may help them to care for patients with serious illness. It may help dispel the misconception that palliative care referral implies imminent death.

If hospital doctors and nurses are to become confident in addressing these difficult issues, they must be assured that they can ask for advice. Informal referrals and corridor discussions are vital, and palliative care staff must be visible on-site at the hospital.

\section{DOUBTS AND DIFFICULTIES}

\section{Financial}

Palliative care services have traditionally been funded through NHS and charitable partnerships and few, if any hospitals, have a budget specifically allocated to palliative care. Data show, however, that acute palliative care units can reduce hospital costs through cost avoidance (unnecessary tests and treatments), by shortening length of stay and facilitating discharge home. ${ }^{27}$

Palliative care is fundamental to hospital care; until there is some re-allocation of funding and facilities to support it, it will continue to depend on private funding. A new Scottish charitable organisation, PATCH (Palliation and the caring hospital), has been established specifically to support hospitals to provide palliative care and ensure that patients are able to access specialist palliative care and symptom control 24 hours a day, seven days a week. It plans to fund Scottish hospitals to develop their palliative care services, and where appropriate, establish dedicated palliative care beds supported by specialist staff.

\section{CONCLUSION}

The concept of specialist beds is not new. Countries without a tradition of community hospice institutions are establishing hospital palliative care units. ${ }^{28}$ Intensive care, high dependency, coronary care, renal dialysis and neonatal care, all have specific medical and nursing expertise for clinical care, teaching and research and the latter is very much needed in palliative care. 
Taking an integrative approach, an acute palliative care unit, designated specialist palliative care beds or a designated ward will not look like a hospital nor will it look like a hospice, it will be something in between. Operating within the hospital organisational structure

\section{REFERENCES}

I Ahmedzai SH, Costa A, Blengini C, (European School of Oncology) et al.A new international framework for palliative care. Eur J Cancer 2004; 40:2 192-200. http://dx.doi.org//0.1016/j.ejca.2004.06.009

2 Ramsay J, National Records of Scotland; Sharpe K, Information Statistics Division. Personal communication of data 2009-12 (2014).

3 Audit Scotland. Review of palliative care services in Scotland [Internet] Edinburgh: Audit Scotland; 2008. Available from: http://www.auditscotland.gov.uk/docs/health/2008/nr_08082I_palliative_care.pdf

4 Gott M, Ingleton C, Bennett MI et al. Transitions to palliative care in acute hospitals in England: qualitative study. BMJ Support Palliat Care 20I I; I:42-8. http://dx.doi.org/I0.I I36/bmj.dI 773

5 Frances R. The Mid Staffordshire NHS Trust Foundation Public Enquiry. London: Stationery Office; 2013.

6 Neuberger J. More care, less pathway. A review of the Liverpool Care Pathway. London: Department of Health; 2013.

7 Information and Statistics Division, Scottish Department of Health. 2009

8 Gomes B, Higginson IJ. Where people die (1974-2030): past trends, future projections and implications for care. Palliat Med 2008; 22:33-4I. http://dx.doi.org//0.II77/02692/6307084606

9 Bloomer MJ, Endacott R, O'Connor M et al. The 'dis-ease' of dying: challenges in nursing care of the dying in the acute hospital setting. A qualitative observational study. Palliat Med 2013; 27:757-64. http://dx.doi.org//0.1 177/02692/63/3477/76

10 Gibbins J, McCoubrie R, Forbes K. Why are newly qualified doctors unprepared to care for patients at the end of life? Med Educ 201I; 45:389-99. http://dx.doi.org//0.1III/j.1365-2923.2010.03873.x

II Greenaway D. Shape of training. Securing the future of excellent patient care [Internet] Shape of Training; 2013. Available from: www.shapeoftraining.co.uk

12 Living and Dying Well Short Life Working Group. Recommendations on palliative care in acute hospitals (2010). Available from: www. scotland.gov.uk/Resource/Doc/924/0105554.doc

13 Clark D, Armstrong M, Allan A et al. Imminence of death among hospital inpatients: prevalent cohort study. Palliat Med 2014; 28: I-6. http://dx.doi.org/I0.II77/02692163/4526443

14 Sigurdardottir KR, Haugen DF. Prevalence of distressing symptoms in hospitalized patients on medical wards: a cross sectional study. BMC Palliat Care 2008; 7:16. http://dx.doi.org//0.1/86//472684X-7-16

15 Gott CM, Ahmedzai SH, Wood C. How many patients in an acute hospital have palliative care needs? Comparing the perspectives of medical and nursing staff. Palliat Med 200I; 15:451-60. http://dx. doi.org/I0.1I91/02692I60I682553932 and with local expertise, it can be a test for palliative care provision and research, a pragmatic approach appropriate to the individual hospital and its patient population. There is a gap in care, and it urgently requires a creative solution.

16 World Health Organization. Cancer pain relief. Geneva:WHO; 1986.

17 Wells M, Dryden H, Guild P et al. The knowledge and attitudes of surgical staff towards the use of opioids in cancer pain management: can the Hospital Palliative Care Team make a difference? Eur J Cancer Care (Engl) 200 I I 0:20 I-I I.http://dx.doi.org/ I 0. I046/ j.1365-2354.200I.00259.x

I8 To TH, Greene AG, Agar MR et al. A point prevalence study of hospital inpatients to define the proportion with palliation as the primary goal of care and the need for specialist palliative care. Intern Med J 20II; 4I:430-3. http://dx.doi. org/I0.IIII/j.I445-5994.20II.02484.x

19 Slaven M,Wylie N, Fitzgerald B et al. Who needs a palliative care consult? The Hamilton chart audit tool.J Palliat Med 2007; 10:3047. http://dx.doi.org//0.1089/jpm.2006.0237

20 The WHO Collaborating Centre for Public Health Palliative Care Programmes. Basic principles, definitions and concepts on the organisation of palliative care programmes [Internet]. The WHO Collaborating Centre for Public Health Palliative Care Programmes; 2009. Available from: http://www.iconcologia.net/catala/qualy/pdf/ whocc_tool_I.pdf

21 Centre to Advance Palliative Care. Public opinion research on palliative care (20I I). New York: 20I I. Available from: http://www.capc.org/ tools-for-palliative-care-programs/marketing/public-opinionresearch/20I I-public-opinion-research-on-palliative-care.pdf

22 Gladwell M. Outliers: the story of success. London: Penguin Books; 2008.

23 Gray $D$ et al. The surgical palliative care ward - an innovative model for the provision of palliative care in an acute hospital. Personal communication, 2014.

24 Paterson F, Buchanan D, Maclvor F et al. Short-stay specialist beds in a UK teaching hospital as a model to integrate palliative care in the acute hospital setting. J R Coll Physicians Edinb 2012; 42:8-14. http://dx.doi.org/I0.4997/JRCPE.2012.103

25 Martin RL. The opposable mind. Winning through integrative thinking. Boston: Harvard Business School Press; 2007.

26 Ballatt J, Campling P. Intelligent kindness: reforming the culture of healthcare. London: RCPsych Publications; 2011.

27 Hanson LC, Usher B, Spragens L et al. Clinical and economic impact of palliative care consultation.J Pain Symptom Manage 2008; 35:340-6. http://dx.doi.org/10.1016/j.jpainsymman.2007.06.008

28 Gomez-Batiste X, Porta-Sales J, Pascual A et al. Catalonia WHO palliative care demonstration project at 15 years.J Pain Symptom Manage 2007; 33:584-90. http://dx.doi.org//0.I0I6/j.jpainsymman.2007.02.019 